\title{
REMARKS ON LOGARITHMICALLY REGULARITY CRITERIA FOR THE 3D VISCOUS MHD EQUATIONS
}

\author{
Xiaochun Chen and Sadek Gala
}

ABSTRACT. In this paper, logarithmically regularity criteria for the 3D MHD equations are established in terms of the Morrey-Camapanto space.

\section{Introduction}

The 3D incompressible viscous MHD equations reads:

$$
\left\{\begin{array}{l}
\partial_{t} u-\mu \Delta u+u \cdot \nabla u+\nabla p+\frac{1}{2} \nabla|b|^{2}-b \cdot \nabla b=0 \\
\partial_{t} b-\nu \Delta b+u \cdot \nabla b-b \cdot \nabla u=0 \\
\nabla \cdot u=\nabla \cdot b=0, \\
u(x, 0)=u_{0}(x), \quad b(x, 0)=b_{0}(x)
\end{array}\right.
$$

where $u=u(x, t) \in \mathbb{R}^{3}$ is the velocity field, $b \in \mathbb{R}^{3}$ is the magnetic field, $p=p(x, t)$ is the scalar pressure, $\mu>0$ is the kinematic viscosity and $\nu>0$ is the resistivity, while $u_{0}$ and $b_{0}$ are given initial velocity and initial magnetic field with $\nabla \cdot u_{0}=\nabla \cdot b_{0}=0$ in the sense of distribution. For simplicity, we assume that the external force has a scalar potential and is included into the pressure gradient. In what follows, we assume $\mu=\nu=1$ for convenience.

It is well-known [11] that the problem (1.1) is local well-posed for any given initial datum $u_{0}, b_{0} \in H^{s}\left(\mathbb{R}^{3}\right), s \geq 3$. But whether this unique local solution can exist globally is an outstanding challenge problem when $n \geq 3$. Some fundamental Serrin's-type regularity criteria in term of the velocity only was done in [5] and [14] independently. Recently, some improvements and extensions were made based on these two basic papers. Part of them are listed here: Chen, Miao and Zhang [3] did improvement in Besov spaces; Zhou and Gala [19] proved regularity for $u$ and $\nabla u$ in the multiplier spaces; $\mathrm{Wu}[13]$ considered the velocity field being in the homogeneous Besov space; regularity was obtained by imposing condition on the pressure in $[15,17]$; in [16] direction of vorticity field $\omega=\nabla \times u$ was discussed (see also [5]).

Received November 17, 2009; Revised February 26, 2010.

2010 Mathematics Subject Classification. 35Q35, 35B65, 76D05.

Key words and phrases. MHD equations, regularity criteria. 
Recently, for the Navier-Stokes equations ( $b=0$ in (1.1)), several log improvements of the Prodi-Serrin criteria were established in $[2,21,18,20]$ in terms of the velocity field.

The purpose of this paper is to establish logarithmically improved regularity criteria in terms of the velocity field or on the gradient of velocity field in terms of the critical Morrey-Camapanato spaces. We will prove:

Theorem 1.1. Let $T>0$ and $\left(u_{0}, b_{0}\right) \in H^{3}\left(\mathbb{R}^{3}\right)$ with $\nabla \cdot u_{0}=\nabla \cdot b_{0}=0$. If the corresponding smooth solution $u(x, t)$ satisfies one of the following conditions

$$
\begin{aligned}
& \int_{0}^{T} \frac{\|u(t, .)\|_{\dot{\mathcal{M}}_{2, \frac{3}{r}}}^{\frac{2}{1-r}}}{1+\ln \left(e+\|u(t, .)\|_{L^{\infty}}\right)} d t<\infty \text { for some } r \text { with } 0<r<1, \\
& \text { (1.3) } \int_{0}^{T} \frac{\|\nabla u(t, .)\|_{\dot{\mathcal{M}}_{2, \frac{3}{r}}}^{\frac{2}{2-r}}}{1+\ln \left(e+\|\nabla u(t, .)\|_{L^{\infty}}\right)} d t \quad<\quad \text { for some } r \text { with } 0<r \leq 1 \text {, }
\end{aligned}
$$

then the smooth solution $(u, b)$ can be extended for $T^{\prime}>T$.

Theorem 1.1 is also true for the 3-D incompressible Navier-Stokes equations, so they gives improvements and extensions of $[2,18,20,21]$.

Remark 1.1. Since the critical Morrey-Campanato space $\dot{\mathcal{M}}_{2, \frac{3}{r}}$ is much wider than the Lebesgue space $L^{\frac{3}{r}}$ and the multiplier space $\dot{X}_{r}$ hence our result covers the recent results given by Zhou and Fan [18].

Remark 1.2. The limiting case $u \in L^{\infty}\left(0, T ; L^{2}\right)$ concerning $(2.1)$ was proved by Seregin [10], by using an approach completely different from energy-type estimate and the proof is based on delicate results on backward uniqueness.

\section{Preliminaries}

Before stating our main result, we recall the definition and some properties of the space that we are going to use. These spaces play an important role in studying the regularity of solutions to partial differential equations (see e.g., [6], [12]).

Definition 2.1. For $1<p \leq q \leq+\infty$, the Morrey-Campanato space $\dot{\mathcal{M}}_{p, q}$ is defined by :

$$
\dot{\mathcal{M}}_{p, q}=\left\{f \in L_{l o c}^{p}\left(\mathbb{R}^{3}\right):\|f\|_{\dot{\mathcal{M}}_{p, q}}=\sup _{x \in \mathbb{R}^{3}} \sup _{R>0} R^{3 / q-3 / p}\|f\|_{L^{p}(B(x, R))}<\infty\right\},
$$

where $B(x, R)$ denotes the ball of center $x$ with radius $R$.

It is easy to verify that $\dot{\mathcal{M}}_{p, q}\left(\mathbb{R}^{3}\right)$ is a Banach space under the norm $\|\cdot\|_{\dot{\mathcal{M}}_{p, q}}$. Furthermore, it is easy to check the following:

$$
\|f(\lambda .)\|_{\dot{\mathcal{M}}_{p, q}}=\frac{1}{\lambda^{\frac{3}{q}}}\|f\|_{\dot{\mathcal{M}}_{p, q}}, \quad \lambda>0 .
$$


Morrey-Campanato spaces can be seen as a complement to $L^{p}$ spaces. In fact, for $p \leq q$, we have

$$
L^{q}=\dot{\mathcal{M}}_{q, q} \subset \dot{\mathcal{M}}_{p, q}
$$

We have the following comparison between Lorentz spaces and MorreyCampanato spaces: for $p \geq 2$,

$$
L^{\frac{3}{r}}\left(\mathbb{R}^{3}\right) \subset L^{\frac{3}{r}, \infty}\left(\mathbb{R}^{3}\right) \subset \dot{\mathcal{M}}_{p, \frac{3}{r}}\left(\mathbb{R}^{3}\right) \subset \dot{X}_{r}\left(\mathbb{R}^{3}\right) \subset \dot{\mathcal{M}}_{2, \frac{3}{r}}\left(\mathbb{R}^{3}\right),
$$

where $L^{p, \infty}$ denotes the usual Lorentz (weak $L^{p}$ ) space.

Due to the following lemma given in [8]:

Lemma 2.2. For $0 \leq r<\frac{3}{2}$, the space $\dot{Z}_{r}$ is defined as the space of $f(x) \in$ $L_{\text {loc }}^{2}\left(\mathbb{R}^{3}\right)$ such that

$$
\|f\|_{\dot{Z}_{r}}=\sup _{\|g\|_{\dot{B}_{2,1}^{r}} \leq 1}\|f g\|_{L^{2}}<\infty
$$

Then $f \in \dot{\mathcal{M}}_{2, \frac{3}{r}}$ if and only if $f \in \dot{Z}_{r}$ with equivalence of norms.

And the fact that

$$
L^{2} \cap \dot{H}^{1} \subset \dot{B}_{2,1}^{r} \subset \dot{H}^{r} \text { for } 0<r<1,
$$

we have

$$
\dot{X}_{r} \subset \dot{\mathcal{M}}_{2, \frac{3}{r}},
$$

where $\dot{X}_{r}$ denotes the point-wise multiplier space from $\dot{H}^{r}$ to $L^{2}$.

We shall prove the following lemma, which will be employed in the proof of our result.

Lemma 2.3. For $0<r<1$, we have

$$
\|f\|_{\dot{B}_{2,1}^{r}} \leq C\|f\|_{L^{2}}^{1-r}\|\nabla f\|_{L^{2}}^{r} .
$$

Proof. The idea comes from [9]. According to the definition of Besov spaces, one has

$$
\begin{aligned}
\|f\|_{\dot{B}_{2,1}^{r}} & =\sum_{j \in \mathbb{Z}} 2^{j r}\left\|\Delta_{j} f\right\|_{L^{2}} \\
& \leq \sum_{j \leq k} 2^{j r}\left\|\Delta_{j} f\right\|_{L^{2}}+\sum_{j>k} 2^{j(r-1)} 2^{j}\left\|\Delta_{j} f\right\|_{L^{2}} \\
& \leq\left(\sum_{j \leq k} 2^{2 j r}\right)^{\frac{1}{2}}\left(\sum_{j \leq k}\left\|\Delta_{j} f\right\|_{L^{2}}^{2}\right)^{\frac{1}{2}}+\left(\sum_{j>k} 2^{2 j(r-1)}\right)^{\frac{1}{2}}\left(\sum_{j>k} 2^{2 j}\left\|\Delta_{j} f\right\|_{L^{2}}^{2}\right)^{\frac{1}{2}} \\
& \leq C\left(2^{r k}\|f\|_{L^{2}}+2^{k(r-1)}\|f\|_{\dot{H}^{1}}\right) \\
& =C\left(2^{r k} A^{-r}+2^{k(r-1)} A^{1-r}\right)\|f\|_{L^{2}}^{1-r}\|f\|_{\dot{H}^{1}}^{r},
\end{aligned}
$$


where $A=\frac{\|f\|_{\dot{H}^{1}}}{\|f\|_{L^{2}}}$. Choose $k$ such that $2^{r k} A^{-r} \leq 1$, that is, $k \leq\left[\log A^{r}\right]$, we thus obtain

$$
\begin{aligned}
\|f\|_{\dot{B}_{2,1}^{r}} & \leq C\left(1+2^{k(r-1)} A^{1-r}\right)\|f\|_{L^{2}}^{1-r}\|f\|_{\dot{H}^{1}}^{r} \\
& \leq C\|f\|_{L^{2}}^{1-r}\|\nabla f\|_{L^{2}}^{r}
\end{aligned}
$$

and so the proof is complete.

\section{Proof of Theorem 1.1}

In order to prove regularity, we need to establish a priori estimates.

Now, we derive estimates under condition (1.2). We follow the argument in [18] and do $H^{1}$ estimates first.

Multiplying the first equation of (1.1) by $\Delta u$, after integration by parts and taking the divergence free property into account, we have

$$
\begin{aligned}
& \frac{1}{2} \frac{d}{d t}\|\nabla u\|_{L^{2}}^{2}+\|\Delta u\|_{L^{2}}^{2} \\
= & -\int_{\mathbb{R}^{3}} \partial_{i} u_{k} \cdot \partial_{k} u_{j} \cdot \partial_{i} u_{j} d x+\int_{\mathbb{R}^{3}} \partial_{i} b_{k} \cdot \partial_{k} b_{j} \cdot \partial_{i} u_{j} d x \\
& -\int_{\mathbb{R}^{3}} b_{k} \cdot \partial_{i} \partial_{k} u_{j} \cdot \partial_{i} b_{j} d x .
\end{aligned}
$$

Similarly, multiplying the second equation of (1.1) by $\Delta b$, we obtain

$$
\begin{aligned}
& \frac{1}{2} \frac{d}{d t}\|\nabla b\|_{L^{2}}^{2}+\|\Delta b\|_{L^{2}}^{2} \\
= & -\int_{\mathbb{R}^{3}} \partial_{i} u_{k} \cdot \partial_{k} b_{j} \cdot \partial_{i} b_{j} d x+\int_{\mathbb{R}^{3}} \partial_{i} b_{k} \cdot \partial_{k} u_{j} \cdot \partial_{i} b_{j} d x \\
& +\int_{\mathbb{R}^{3}} b_{k} \cdot \partial_{k} \partial_{i} u_{j} \cdot \partial_{i} b_{j} d x .
\end{aligned}
$$

Combining (3.1) and (3.2) (cancellation happens) yields

$$
\begin{aligned}
& \frac{1}{2} \frac{d}{d t}\left(\|\nabla u\|_{L^{2}}^{2}+\|\nabla b\|_{L^{2}}^{2}\right)+\|\Delta u\|_{L^{2}}^{2}+\|\Delta b\|_{L^{2}}^{2} \\
= & -\int_{\mathbb{R}^{3}} \partial_{i} u_{k} \cdot \partial_{k} u_{j} \cdot \partial_{i} u_{j} d x+\int_{\mathbb{R}^{3}} \partial_{i} b_{k} \cdot \partial_{k} b_{j} \cdot \partial_{i} u_{j} d x \\
& -\int_{\mathbb{R}^{3}} \partial_{i} u_{k} \cdot \partial_{k} b_{j} \cdot \partial_{i} b_{j} d x+\int_{\mathbb{R}^{3}} \partial_{i} b_{k} \cdot \partial_{k} u_{j} \cdot \partial_{i} b_{j} d x .
\end{aligned}
$$

Taking integration parts on (3.3) once to taking $u$ out as

$$
\begin{aligned}
& \frac{1}{2} \frac{d}{d t}\left(\|\nabla u\|_{L^{2}}^{2}+\|\nabla b\|_{L^{2}}^{2}\right)+\|\Delta u\|_{L^{2}}^{2}+\|\Delta b\|_{L^{2}}^{2} \\
= & \int_{\mathbb{R}^{3}} u_{k} \cdot \partial_{i}\left(\partial_{k} u_{j} \cdot \partial_{i} u_{j}\right) d x-\int_{\mathbb{R}^{3}} \partial_{i}\left(\partial_{i} b_{k} \cdot \partial_{k} b_{j}\right) \cdot u_{j} d x \\
& +\int_{\mathbb{R}^{3}} u_{k} \cdot \partial_{i}\left(\partial_{k} b_{j} \cdot \partial_{i} b_{j}\right) d x-\int_{\mathbb{R}^{3}} \partial_{k}\left(\partial_{i} b_{k} \cdot \partial_{i} b_{j}\right) \cdot u_{j} d x
\end{aligned}
$$




$$
=I+I I+I I I+I V .
$$

We do estimate for $I+I I+I I I+I V$ by Hölder's inequality and Young's inequality firstly as

$$
\begin{aligned}
& |I+I I+I I I+I V| \\
\leq & C\|u\|_{\mathcal{M}_{2, \frac{3}{r}}}\|\nabla u\|_{\dot{B}_{2,1}^{r}}\|\Delta u\|_{L^{2}}+C\|u\|_{\mathcal{M}_{2, \frac{3}{r}}}\|\nabla b\|_{\dot{B}_{2,1}^{r}}\|\Delta b\|_{L^{2}} \\
\leq & C\|u\|_{\mathcal{M}_{2, \frac{3}{r}}}\|\nabla u\|_{L^{2}}^{1-r}\|\Delta u\|_{L^{2}}^{1+r}+C\|u\|_{\mathcal{M}_{2, \frac{3}{r}}}^{1+r}\|\nabla b\|_{L^{2}}^{1-r}\|\Delta b\|_{L^{2}}^{1+r} \\
\leq & C\|u\|_{\mathcal{M}_{2, \frac{3}{r}}^{1-r}}^{\frac{2}{1-r}}\left(\|\nabla u\|_{L^{2}}^{2}+\|\nabla b\|_{L^{2}}^{2}\right)+\frac{1}{2}\left(\|\Delta u\|_{L^{2}}^{2}+\|\Delta b\|_{L^{2}}^{2} .\right.
\end{aligned}
$$

For the first term in the right hand side of (3.5), we have

$$
\begin{aligned}
& \|u\|_{\mathcal{\mathcal { M }}_{2, \frac{3}{r}}}^{\frac{2}{1-r}}\left(\|\nabla u\|_{L^{2}}^{2}+\|\nabla b\|_{L^{2}}^{2}\right) \\
\leq & \frac{\|u\|_{\mathcal{\mathcal { M }}_{2, \frac{3}{r}}}^{\frac{2}{1-r}}}{1+\ln \left(e+\|u\|_{\left.L^{\infty}\right)}\right.}\left(\|\nabla u\|_{L^{2}}^{2}+\|\nabla b\|_{L^{2}}^{2}\right)\left(1+\ln \left(e+\|u\|_{L^{\infty}}\right)\right) \\
\leq & C \frac{\|u\|_{\mathcal{M}_{2, \frac{3}{r}}}^{\frac{2}{1-r}}}{1+\ln \left(e+\|u\|_{L^{\infty}}\right)}\left(\|\nabla u\|_{L^{2}}^{2}+\|\nabla b\|_{L^{2}}^{2}\right)\left(1+\ln \left(e+\left\|\nabla^{3} u\right\|_{L^{2}}+\left\|\nabla^{3} b\right\|_{L^{2}}\right)\right),
\end{aligned}
$$

where Sobolev embedding was used.

For any $T_{0}<t \leq T$, we let

$$
y(t):=\sup _{T_{0} \leq s \leq t}\left(\left\|\nabla^{3} u\right\|_{L^{2}}+\left\|\nabla^{3} b\right\|_{L^{2}}\right) .
$$

Coming back to (3.4), we get

$$
\begin{aligned}
& \frac{d}{d t}\left(\|\nabla u\|_{L^{2}}^{2}+\|\nabla b\|_{L^{2}}^{2}\right)+\|\Delta u\|_{L^{2}}^{2}+\|\Delta b\|_{L^{2}}^{2} \\
\leq & C \frac{\|u\|_{\mathcal{M}_{2, \frac{3}{r}}}^{\frac{2}{1-r}}}{1+\ln \left(e+\|u\|_{\left.L^{\infty}\right)}\right.}\left(\|\nabla u\|_{L^{2}}^{2}+\|\nabla b\|_{L^{2}}^{2}\right)(1+\ln (e+y(t))) .
\end{aligned}
$$

Applying Gronwall's inequality on (3.7) for the interval $\left[T_{0}, t\right]$, one has

$$
\begin{aligned}
& \|\nabla u(., t)\|_{L^{2}}^{2}+\|\nabla b(., t)\|_{L^{2}}^{2} \\
\leq & \left(\left\|\nabla u\left(., T_{0}\right)\right\|_{L^{2}}^{2}+\left\|\nabla u\left(., T_{0}\right)\right\|_{L^{2}}^{2}\right) \\
& \times \exp \left(C(1+\ln (e+y(t))) \int_{T_{0}}^{t} \frac{\|u\|_{\mathcal{M}_{2, \frac{3}{r}}}^{\frac{2}{1-r}}}{1+\ln \left(e+\|u\|_{L^{\infty}}\right)} d s\right) \\
\leq & C_{0} \exp (C \epsilon(1+\ln (e+y(t)))) \leq C_{0} \exp (2 C \epsilon \ln (e+y(t))) \\
\leq & C_{0}(e+y(t))^{2 C \epsilon},
\end{aligned}
$$


provided that

$$
\int_{T_{0}}^{t} \frac{\|u\|_{\dot{\mathcal{M}}_{2, \frac{3}{r}}}^{\frac{2}{1-r}}}{1+\ln \left(e+\|u\|_{L^{\infty}}\right)} d s<\epsilon \ll 1,
$$

and where $C_{0}=\left\|\nabla u\left(., T_{0}\right)\right\|_{L^{2}}^{2}+\left\|\nabla u\left(., T_{0}\right)\right\|_{L^{2}}^{2}$.

Then we go to the estimate for $H^{3}$ norm. Taking the operation $\Lambda^{3}=(-\Delta)^{\frac{3}{2}}$ on both sides of (1.1), then multiplying them by $\Lambda^{3} u$ and $\Lambda^{3} b$ respectively, after integrating over $\mathbb{R}^{3}$, we have

$$
\begin{aligned}
& \frac{1}{2} \frac{d}{d t}\left(\left\|\Lambda^{3} u(t)\right\|_{L^{2}}^{2}+\left\|\Lambda^{3} b(t)\right\|_{L^{2}}^{2}\right)+\left\|\Lambda^{3} \nabla u(t)\right\|_{L^{2}}^{2}+\left\|\Lambda^{3} \nabla b(t)\right\|_{L^{2}}^{2} \\
= & -\int_{\mathbb{R}^{3}} \Lambda^{3}(u \cdot \nabla u) \Lambda^{3} u d x+\int_{\mathbb{R}^{3}} \Lambda^{3}(b \cdot \nabla b) \Lambda^{3} u d x \\
& -\int_{\mathbb{R}^{3}} \Lambda^{3}(u \cdot \nabla b) \Lambda^{3} b d x+\int_{\mathbb{R}^{3}} \Lambda^{3}(b \cdot \nabla u) \Lambda^{3} b d x .
\end{aligned}
$$

Noting that $\nabla \cdot u=\nabla \cdot b=0$ and integrating by parts, we write (3.9) as

$$
\begin{aligned}
& \frac{1}{2} \frac{d}{d t}\left(\left\|\Lambda^{3} u(t)\right\|_{L^{2}}^{2}+\left\|\Lambda^{3} b(t)\right\|_{L^{2}}^{2}\right)+\left\|\Lambda^{3} \nabla u(t)\right\|_{L^{2}}^{2}+\left\|\Lambda^{3} \nabla b(t)\right\|_{L^{2}}^{2} \\
= & -\int_{\mathbb{R}^{3}}\left[\Lambda^{3}(u \cdot \nabla u)-u \cdot \Lambda^{3} \nabla u\right] \Lambda^{3} u d x-\int_{\mathbb{R}^{3}}\left[\Lambda^{3}(u \cdot \nabla b)-u \cdot \Lambda^{3} \nabla b\right] \Lambda^{3} b d x \\
& +\int_{\mathbb{R}^{3}}\left[\Lambda^{3}(b \cdot \nabla b)-b \cdot \Lambda^{3} \nabla b\right] \Lambda^{3} u d x+\int_{\mathbb{R}^{3}}\left[\Lambda^{3}(b \cdot \nabla u)-b \cdot \Lambda^{3} \nabla u\right] \Lambda^{3} b d x \\
= & \Pi_{1}+\Pi_{2}+\Pi_{3}+\Pi_{4} .
\end{aligned}
$$

In what follows, we will use the following inequality due to Kenig, Ponce and Vega [7]:

(3.11) $\left\|\Lambda^{\alpha}(f g)-f \Lambda^{\alpha} g\right\|_{L^{p}} \leq C\left(\left\|\Lambda^{\alpha-1} g\right\|_{L^{q_{1}}}\|\nabla f\|_{L^{p_{1}}}+\left\|\Lambda^{\alpha} f\right\|_{L^{p_{2}}}\|g\|_{L^{q_{2}}}\right)$, for $\alpha>1$, and $\frac{1}{p}=\frac{1}{p_{1}}+\frac{1}{q_{1}}=\frac{1}{p_{2}}+\frac{1}{q_{2}}$.

Hence $\Pi_{1}$ can be estimated as

$$
\begin{aligned}
\Pi_{1} & \leq C\|\nabla u\|_{L^{3}}\left\|\Lambda^{3} u\right\|_{L^{3}}^{2} \leq C\|\nabla u\|_{L^{2}}^{\frac{13}{12}}\left\|\Lambda^{3} u\right\|_{L^{2}}^{\frac{1}{4}}\left\|\Lambda^{4} u\right\|_{L^{2}}^{\frac{5}{3}} \\
& \leq \frac{1}{6}\left\|\Lambda^{4} u\right\|_{L^{2}}^{2}+C\|\nabla u\|_{L^{2}}^{\frac{13}{2}}\left\|\Lambda^{3} u\right\|_{L^{2}}^{\frac{3}{2}}
\end{aligned}
$$

where we used (3.11) with $\alpha=3, p=\frac{3}{2}, p_{1}=q_{1}=p_{2}=q_{2}=3$, and the following inequalities

$$
\|\nabla u\|_{L^{3}} \leq C\|\nabla u\|_{L^{2}}^{\frac{3}{4}}\left\|\Lambda^{3} u\right\|_{L^{2}}^{\frac{1}{4}},
$$

and

$$
\left\|\Lambda^{3} u\right\|_{L^{3}} \leq C\|\nabla u\|_{L^{2}}^{\frac{1}{6}}\left\|\Lambda^{4} u\right\|_{L^{2}}^{\frac{5}{6}} .
$$


If we use the existing estimate (3.8) for $T_{0}<t<T$, (3.12) reduces to

$$
\Pi_{1} \leq \frac{1}{6}\left\|\Lambda^{4} u\right\|_{L^{2}}^{2}+C_{0} C(e+y(t))^{\frac{3}{2}+\frac{13}{2} C \epsilon} .
$$

Similarly, we can do estimate for $\Pi_{3}$ as

$$
\begin{aligned}
\Pi_{3} & \leq\left\|\Lambda^{3}(b \cdot \nabla b)-b \cdot \Lambda^{3} \nabla b\right\|_{L^{\frac{3}{2}}}\left\|\Lambda^{3} u\right\|_{L^{3}} \\
& \leq C\|\nabla b\|_{L^{3}}\left\|\Lambda^{3} b\right\|_{L^{3}}\left\|\Lambda^{3} u\right\|_{L^{3}} \\
& \leq C\left(\|\nabla b\|_{L^{2}}^{\frac{3}{4}}\left\|\Lambda^{3} b\right\|_{L^{2}}^{\frac{1}{4}}\right)\left(\|\nabla b\|_{L^{2}}^{\frac{1}{6}}\left\|\Lambda^{4} b\right\|_{L^{2}}^{\frac{5}{6}}\right)\left(\|\nabla u\|_{L^{2}}^{\frac{1}{6}}\left\|\Lambda^{4} u\right\|_{L^{2}}^{\frac{5}{6}}\right) \\
& \leq C\|\nabla b\|_{L^{2}}^{\frac{3}{4}}\left\|\Lambda^{3} b\right\|_{L^{2}}^{\frac{1}{4}}\left(\|\nabla u\|_{L^{2}}+\|\nabla b\|_{L^{2}}\right)^{\frac{1}{3}}\left(\left\|\Lambda^{4} u\right\|_{L^{2}}+\left\|\Lambda^{4} b\right\|_{L^{2}}\right)^{\frac{5}{3}} \\
& \leq C\left(\|\nabla u\|_{L^{2}}+\|\nabla b\|_{L^{2}}\right)^{\frac{1}{3}+\frac{3}{4}}\left\|\Lambda^{3} b\right\|_{L^{2}}^{\frac{1}{4}}\left(\left\|\Lambda^{4} u\right\|_{L^{2}}+\left\|\Lambda^{4} b\right\|_{L^{2}}\right)^{\frac{5}{3}} \\
& \leq \frac{1}{6}\left(\left\|\Lambda^{4} u\right\|_{L^{2}}^{2}+\left\|\Lambda^{4} b\right\|_{L^{2}}^{2}\right)+C\left(\|\nabla u\|_{L^{2}}+\|\nabla b\|_{L^{2}}\right)^{\frac{13}{2}}\left\|\Lambda^{3} b\right\|_{L^{2}}^{\frac{3}{2}} \\
(3.16) & \leq \frac{1}{6}\left(\left\|\Lambda^{4} u\right\|_{L^{2}}^{2}+\left\|\Lambda^{4} b\right\|_{L^{2}}^{2}\right)+C_{0} C(e+y(t))^{\frac{3}{2}+\frac{13}{2} C \epsilon} .
\end{aligned}
$$

Using (3.13) and (3.14) again, we have

$$
\begin{aligned}
\Pi_{2}+\Pi_{4} & \leq C\left(\|\nabla b\|_{L^{3}}\left\|\Lambda^{3} u\right\|_{L^{3}}+\|\nabla u\|_{L^{3}}\left\|\Lambda^{3} b\right\|_{L^{3}}\right)\left\|\Lambda^{3} b\right\|_{L^{3}} \\
& \leq C\left(\|\nabla b\|_{L^{3}}+\|\nabla u\|_{L^{3}}\right)\left(\left\|\Lambda^{3} b\right\|_{L^{3}}^{2}+\left\|\Lambda^{3} u\right\|_{L^{3}}^{2}\right) \\
& \leq \frac{1}{6}\left(\left\|\Lambda^{4} u\right\|_{L^{2}}^{2}+\left\|\Lambda^{4} b\right\|_{L^{2}}^{2}\right)+C_{0} C(e+y(t))^{\frac{3}{2}+\frac{13}{2} C \epsilon} .
\end{aligned}
$$

Combining (3.10), (3.15), (3.16) and (3.17), we easily get

$$
\frac{d}{d t}\left(\left\|\Lambda^{3} u(t)\right\|_{L^{2}}^{2}+\left\|\Lambda^{3} b(t)\right\|_{L^{2}}^{2}\right) \leq C_{0} C(e+y(t))^{\frac{3}{2}+\frac{13}{4} C \epsilon} .
$$

Gronwall's inequality implies the boundness of $H^{3}$-norm of $u$ and $b$ provided that $\epsilon<\frac{1}{13 C}$, which can be achieved by the absolute continuous property of integral (1.2).

This completes the proof under condition (1.2).

Then, we go to the proof for Theorem 1.1 under (1.3). First, we assume $0<r<1$. We start from (3.3),

$$
\begin{aligned}
& \frac{1}{2} \frac{d}{d t}\left(\|\nabla u\|_{L^{2}}^{2}+\|\nabla b\|_{L^{2}}^{2}\right)+\|\Delta u\|_{L^{2}}^{2}+\|\Delta b\|_{L^{2}}^{2} \\
= & -\int_{\mathbb{R}^{3}} \partial_{i} u_{k} \cdot \partial_{k} u_{j} \cdot \partial_{i} u_{j} d x+\int_{\mathbb{R}^{3}} \partial_{i} b_{k} \cdot \partial_{k} b_{j} \cdot \partial_{i} u_{j} d x \\
& -\int_{\mathbb{R}^{3}} \partial_{i} u_{k} \cdot \partial_{k} b_{j} \cdot \partial_{i} b_{j} d x+\int_{\mathbb{R}^{3}} \partial_{i} b_{k} \cdot \partial_{k} u_{j} \cdot \partial_{i} b_{j} d x \\
\leq & C\|\nabla u\|_{\dot{\mathcal{M}}_{2, \frac{3}{r}}}\|\nabla u\|_{\dot{B}_{2,1}^{r}}\|\nabla u\|_{L^{2}}+C\|\nabla u\|_{\dot{\mathcal{M}}_{2, \frac{3}{r}}}\|\nabla b\|_{\dot{B}_{2,1}^{r}}\|\nabla b\|_{L^{2}} \\
\leq & C\|\nabla u\|_{\dot{\mathcal{M}}_{2, \frac{3}{r}}}\left(\|\nabla u\|_{L^{2}}^{2-r}\|\Delta u\|_{L^{2}}^{r}+\|\nabla b\|_{L^{2}}^{2-r}\|\Delta b\|_{L^{2}}^{r}\right)
\end{aligned}
$$




$$
\leq \frac{1}{2}\left(\|\Delta u\|_{L^{2}}^{2}+\|\Delta b\|_{L^{2}}^{2}\right)+C\|\nabla u\|_{\dot{\mathcal{M}}_{2, \frac{3}{r}}}^{\frac{2}{2-r}}\left(\|\nabla u\|_{L^{2}}^{2}+\|\nabla b\|_{L^{2}}^{2}\right) .
$$

Therefore, we have

$$
\begin{aligned}
& \frac{d}{d t}\left(\|\nabla u\|_{L^{2}}^{2}+\|\nabla b\|_{L^{2}}^{2}\right) \\
\leq & C\|\nabla u\|_{\dot{\mathcal{M}}_{2, \frac{3}{r}}^{2-r}}^{\frac{2}{2-r}}\left(\|\nabla u\|_{L^{2}}^{2}+\|\nabla b\|_{L^{2}}^{2}\right) \\
\leq & C \frac{\|\nabla u\|_{\mathcal{M}_{2, \frac{3}{r}}^{2-r}}^{\frac{2}{2-r}}}{1+\ln \left(e+\|\nabla u\|_{L^{\infty}}\right)}\left(\|\nabla u\|_{L^{2}}^{2}+\|\nabla b\|_{L^{2}}^{2}\right)\left(1+\ln \left(e+\|\nabla u\|_{L^{\infty}}\right)\right) \\
\leq & C \frac{\|\nabla u\|_{\dot{\mathcal{M}}_{2, \frac{3}{r}}}^{\frac{2}{2-r}}}{1+\ln \left(e+\|\nabla u\|_{L^{\infty}}\right)}\left(\|\nabla u\|_{L^{2}}^{2}+\|\nabla b\|_{L^{2}}^{2}\right)(1+\ln (e+y(t))),
\end{aligned}
$$

where $y(t)$ is defined by (3.6).

Applying Gronwall's inequality on (3.18) for the interval $\left[T_{0}, t\right]$, one has

$$
\begin{aligned}
\|\nabla u(., t)\|_{L^{2}}^{2}+\|\nabla b(., t)\|_{L^{2}}^{2} & \leq C_{0} \exp (C \epsilon(1+\ln (e+y(t)))) \\
& \leq C_{0} \exp (2 C \epsilon \ln (e+y(t))) \\
& \leq C_{0}(e+y(t))^{2 C \epsilon},
\end{aligned}
$$

provided that

$$
\int_{T_{0}}^{t} \frac{\|\nabla u\|_{\dot{\mathcal{M}}_{2, \frac{3}{r}}}^{\frac{2}{2-r}}}{1+\ln \left(e+\|\nabla u\|_{L^{\infty}}\right)} d s<\epsilon \ll 1,
$$

and where $C_{0}=\left\|\nabla u\left(., T_{0}\right)\right\|_{L^{2}}^{2}+\left\|\nabla u\left(., T_{0}\right)\right\|_{L^{2}}^{2}$.

From (3.19), $H^{3}$ estimate for this case is same as that for the first case.

The proof is complete.

When $r=1$ in (1.3), We need the following lemma:

Lemma 3.1. If $f \in H^{1}\left(\mathbb{R}^{3}\right)$ and $\nabla f \in \dot{\mathcal{M}}_{2,3}\left(\mathbb{R}^{3}\right)$, then

$$
f \in B M O\left(\mathbb{R}^{3}\right) .
$$

Proof. By the classical Poincaré inequality, we have

$$
\begin{aligned}
\int_{B(x, R)}\left|f(y)-f_{B(x, R)}\right|^{2} d y & \leq C R^{2} \int_{B(x, R)}|\nabla f(y)|^{2} d y \\
& \leq C R^{3}\|\nabla f\|_{\mathcal{M}_{2,3}}^{2}
\end{aligned}
$$

for every ball $B(x, R)$ of any radius $R$ and there holds

$$
\begin{aligned}
\|f\|_{B M O}^{2} & =\sup _{x \in \mathbb{R}^{3}} \sup _{R>0} \frac{1}{|B(x, R)|} \int_{B(x, R)}\left|f(y)-f_{B(x, R)} f(y)\right|^{2} d y \\
& \leq C\|\nabla f\|_{\dot{\mathcal{M}}_{2,3}}^{2} .
\end{aligned}
$$


Since $\nabla \cdot w=0$, it follows from Coifman-Lions-Meyer-Semmes [1] (see also [4]) that

$$
w \cdot \nabla w \in \mathcal{H}^{1} \text { with }\|w \cdot \nabla w\|_{\mathcal{H}^{1}} \leq C\|\nabla w\|_{L^{2}}\|w\|_{L^{2}},
$$

where $\mathcal{H}^{1}$ denotes the Hardy space on $\mathbb{R}^{3}$. Since $\left(\mathcal{H}^{1}\right)^{*}=B M O,(3.5)$ reads

$$
\begin{aligned}
& |I+I I+I I I+I V| \\
\leq & C\|u\|_{B M O}\|\nabla u\|_{L^{2}}\|\Delta u\|_{L^{2}}+C\|u\|_{B M O}\|\nabla b\|_{L^{2}}\|\Delta b\|_{L^{2}} \\
\leq & C\|u\|_{\mathcal{M}_{2,3}}\|\nabla u\|_{L^{2}}\|\Delta u\|_{L^{2}}+C\|u\|_{\mathcal{M}_{2,3}}\|\nabla b\|_{L^{2}}\|\Delta b\|_{L^{2}} \\
\leq & C\|\nabla u\|_{\mathcal{M}_{2,3}}^{2}\left(\|\nabla u\|_{L^{2}}^{2}+\|\nabla b\|_{L^{2}}^{2}\right)+\frac{1}{2}\left(\|\Delta u\|_{L^{2}}^{2}+\|\Delta b\|_{L^{2}}^{2}\right) .
\end{aligned}
$$

Then (3.8) reduces to

$$
\begin{aligned}
& \frac{d}{d t}\left(\|\nabla u\|_{L^{2}}^{2}+\|\nabla b\|_{L^{2}}^{2}\right)+\|\Delta u\|_{L^{2}}^{2}+\|\Delta b\|_{L^{2}}^{2} \\
\leq & C \frac{\|u\|_{\mathcal{M}_{2,3}}^{2}}{1+\ln \left(e+\|u\|_{L^{\infty}}\right)}\left(\|\nabla u\|_{L^{2}}^{2}+\|\nabla b\|_{L^{2}}^{2}\right)(1+\ln (e+y(t))) .
\end{aligned}
$$

The remaining estimate is analogous to that for $r<1$.

Acknowledgment. The authors would like to thank the referee for his/her useful comments and suggestions. The authors are deeply thankful to Professor Yong Zhou for helpful discussions, suggestions and constant encouragement.

\section{References}

[1] R. Coifman, P. L. Lions, Y. Meyer, and S. Semmes, Compensated compactness and Hardy spaces, J. Math. Pures Appl. (9) 72 (1993), no. 3, 247-286.

[2] C. H. Chan and A. Vasseur, Log improvement of the Prodi-Serrin criteria for NavierStokes equations, Methods Appl. Anal. 14 (2007), no. 2, 197-212.

[3] Q. Chen, C. Miao, and Z. Zhang, On the regularity criterion of weak solution for the $3 D$ viscous magneto-hydrodynamics equations, Comm. Math. Phys. 284 (2008), no. 3, 919-930.

[4] S. Gala, A note on div-curl lemma, Serdica Math. J. 33 (2007), no. 2-3, 339-350.

[5] C. He and Z. Xin, On the regularity of weak solutions to the magnetohydrodynamic equations, J. Differential Equations 213 (2005), no. 2, 235-254.

[6] T. Kato, Strong solutions of the Navier-Stokes equation in Morrey spaces, Bol. Soc. Brasil. Mat. (N.S.) 22 (1992), no. 2, 127-155.

[7] C. Kenig, G. Ponce, and L. Vega, Well-posedness of the initial value problem for the Korteweg-de Vries equation, J. Amer. Math. Soc. 4 (1991), no. 2, 323-347.

[8] P. G. Lemarié-Rieusset, The Navier-Stokes equations in the critical Morrey-Campanato space, Rev. Mat. Iberoam. 23 (2007), no. 3, 897-930.

[9] S. Machihara and T. Ozawa, Interpolation inequalities in Besov spaces, Proc. Amer. Math. Soc. 131 (2003), no. 5, 1553-1556.

[10] G. A. Seregin, A new version of the Ladyzhenskaya-Prodi-Serrin condition, Algebra i Analiz 18 (2006), no. 1, 124-143; translation in St. Petersburg Math. J. 18 (2007), no. $1,89-103$.

[11] M. Sermange and R. Temam, Some mathematical questions related to the MHD equations, Comm. Pure Appl. Math. 36 (1983), no. 5, 635-664. 
[12] M. E. Taylor, Analysis on Morrey spaces and applications to Navier-Stokes and other evolution equations, Comm. Partial Differential Equations 17 (1992), no. 9-10, 14071456.

[13] J. Wu, Regularity criteria for the generalized MHD equations, Comm. Partial Differential Equations 33 (2008), no. 1-3, 285-306.

[14] Y. Zhou, Remarks on regularities for the 3D MHD equations, Discrete Contin. Dyn. Syst. 12 (2005), no. 5, 881-886.

[15] - Regularity criteria for the 3D MHD equations in terms of the pressure, Internat. J. Non-Linear Mech. 41 (2006), no. 10, 1174-1180.

[16] - Regularity criteria for the generalized viscous MHD equations, Ann. Inst. H. Poincaré Anal. Non Linéaire 24 (2007), no. 3, 491-505.

[17] Y. Zhou and J. Fan, On regularity criteria in terms of pressure for the 3D viscous MHD equations, Unpublished, 2008.

[18] _ Logarithmically improved regularity criteria for the 3-D viscous MHD equations, Submitted, 2009.

[19] Y. Zhou and S. Gala, Regularity criteria for the solutions to the 3D MHD equations in the multiplier space, Z. Angew. Math. Phys. 61 (2010), no. 2, 193-199.

[20] _ Logarithmically improved regularity criteria for the Navier-Stokes equations in multiplier spaces, J. Math. Anal. Appl. 356 (2009), no. 2, 498-501.

[21] Y. Zhou and Z. Lei, Logarithmically improved criteria for Euler and Navier-Stokes equations, Preprint 2008.

Xiaochun Chen

Department of Mathematics

Chonging Three Gorges University

Wanzhou 404000, Chongling, P. R. China

E-mail address: xiaochunchen1@gmail.com

SADEK GALA

Department of Mathematics

University of MOSTAGANEM

Box 227, Mostaganem 27000, Algeria

E-mail address: sadek.gala@gmail.com 